\title{
ETA-PRODUCTS WHICH ARE SIMULTANEOUS EIGENFORMS OF HECKE OPERATORS
}

by ANTHONY J. F. BIAGIOLI

(Received 25 November 1991)

1. Statement of results. The Dedekind eta-function is defined for any $\tau$ in the upper half-plane by

$$
\eta(\tau)=x^{1 / 24} \prod_{n=1}^{\infty}\left(1-x^{n}\right),
$$

where $x=\exp (2 \pi i \tau)$ and $x^{1 / 24}=\exp (2 \pi i \tau / 24)$. By an eta-product we shall mean a function

$$
f(\tau)=\prod_{\delta \mid N} \eta(\delta \tau)^{r_{s}}
$$

where $N \geq 1$ and each $r_{\delta} \in \mathbb{Z}$. In addition, we shall always assume that $k=\frac{1}{2} \sum_{\delta \mid N} r_{\delta}$ is an integer. Using the Legendre-Jacobi symbol (-), we define a Dirichlet character $\epsilon$ by

$$
\epsilon(a)=\left(\frac{(-1)^{k}}{a}\right) \prod_{\delta \mid N}\left(\frac{\delta}{a}\right)^{r_{s}}
$$

when $a$ is odd. If $p$ is a prime for which $\epsilon(p) \neq 0$ and if $F$ is a function with a Fourier series

$$
F(\tau)=\sum_{m \geq m_{0}} a(m) x^{m},
$$

then we define a Hecke operator $T_{p}$ by

where

$$
F\left|T_{p}=F\right| U_{p}+\epsilon(p) F \mid V_{p}
$$

and

$$
F \mid U_{p}=\sum_{p m \geq m_{0}} a(p m) x^{m}
$$

$$
F \mid V_{p}=p^{k-1} \sum_{m \geq m_{11}} a(m) x^{p m}
$$

The function $f(\tau)$ is a modular form of weight $k$ on the group $\Gamma_{0}(N)$ and at any cusp $a / c \in \mathbb{Q} \cup\{\infty\}$ it has order

$$
\operatorname{Ord}_{\Gamma_{\mathrm{u}}(N)}\left(f ; \frac{a}{c}\right)=\frac{C(d)}{24}
$$

where $d=(N, c)$ and

$$
C(d)=\frac{N}{\left(N, d^{2}\right)} \sum_{\delta \mid N} \frac{(d, \delta)^{2}}{\delta} r_{\delta}
$$

Glasgow Math. J. 35 (1993) 307-323. 
We have $d \mid N$ and so $C(d)$ is always an integer. We define the ramification index of $f$ as $\zeta=a / c$ to be the positive integer $e_{\zeta}$ determined by reducing the fraction

$$
\frac{C(d)}{24}=\frac{c_{\zeta}}{e_{\zeta}}
$$

to lowest terms. Thus $c_{\zeta}=C(d) /(24, C(d))$ and $e_{\zeta}=24 /(24, C(d))$. In particular we denote $e_{\infty}$ by $e$ and $c_{\infty}$ by $c$. We let $E$ denote the least common multiple of all the ramification indices of $f$; since $e_{\zeta}$ depends only on $d$ we thus have

$$
E=\text { l.c.m. }\left\{e_{1 / d}: d \mid N\right\}
$$

and this is a divisor of 24 .

The principal objective of this paper is the following

THEOREM. Let $F(\tau)=f(e \tau)$ and suppose

$$
0 \leq C(d) \leq 24 \text { for every } d \mid N \text {. }
$$

Then $F$ is a simultaneous eigenform for the family of Hecke operators

$$
\left\{T_{p}: p \equiv 1(\bmod E) \text { and } p \nmid N\right\} .
$$

This was proven for $N$ prime by Morris Newman [12] and for $N=4$ by Gordon and Sinor [6].

2. Some background. We let $\mathbb{Z}, \mathbb{Q}, \mathbb{C}, \mathbb{H}$ denote the integers, the rational numbers, the complex numbers, and the complex upper half-plane, respectively, we let infinity be represented as $\infty=1 / 0$, and we set $\mathbb{Q}^{*}=\mathbb{Q} \cup\{\infty\}, \mathbb{C}^{*}=\mathbb{C} \cup\{\infty\}$, and $0 \mathbb{-}^{*}=\mathbb{Q} \cup \cup \mathbb{Q} \cup\{\infty\}$. For any $A=\left(\begin{array}{ll}a & b \\ c & d\end{array}\right) \in M_{2}^{+}(\mathbb{Z})$, the set of $2 \times 2$ integral matrices with positive determinant, and any function $f: \mathbb{H} \mathbb{H}^{*} \rightarrow \mathbb{C}^{*}$ we define $f \circ A: \mathbb{\mathbb { H } ^ { * }} \rightarrow \mathbb{C}^{*}$ by

$$
f \circ A(\tau)=f\left(\frac{a \tau+b}{c \tau+d}\right) .
$$

When $n$ is a positive integer we use the abbreviation

$$
f \circ n=f \circ\left(\begin{array}{ll}
n & 0 \\
0 & 1
\end{array}\right) .
$$

The stroke operator of weight $k$ is defined for any real number $k$ by $f \mapsto f \mid A$, where $f \mid A: \mathbb{A}^{*} \rightarrow \mathbb{C}^{*}$ is the function

$$
f \mid A(\tau)=(\operatorname{det} M)^{+k / 2}(c \tau+d)^{-k} f \circ A(\tau) .
$$

We shall determine the power in $(c \tau+d)^{-k}$ by taking the argument of a complex number $z$ in the range $-\pi<\arg (z) \leq \pi$.

Some subgroups of $M_{2}^{+}(\mathbb{Z})$ we shall work with are the modular group,

$$
\Gamma(1)=\left\{A \in M_{2}^{+}(\mathbb{Z}): \operatorname{det} A=1\right\},
$$


the principal congruence subgroup of level $N$,

$$
\Gamma(N)=\left\{A \in \Gamma(1): A \equiv \pm\left(\begin{array}{ll}
1 & 0 \\
0 & 1
\end{array}\right) \quad(\bmod N)\right\}
$$

the principal transform subgroup of level $N$

$$
\Gamma_{0}(N)=\left\{\left(\begin{array}{ll}
a & b \\
c & d
\end{array}\right) \in \Gamma(1): c \equiv 0 \quad(\bmod N)\right\}
$$

and the related subgroup

$$
\Gamma_{0}\left(N_{1}, N_{2}\right)=\left\{\left(\begin{array}{ll}
a & b \\
c & d
\end{array}\right) \in \Gamma(1): N_{1} \mid c \text { and } N_{2} \mid b\right\} .
$$

If $f$ is a modular form on a subgroup $\Gamma \leq \Gamma(1)$ of finite index, we define an order function $\operatorname{ord}(f ; \zeta)$ at any cusp $\zeta \in \mathbb{Q}^{*}$ by

$$
\operatorname{ord}(f ; \zeta)=\frac{1}{\kappa(\Gamma ; \zeta)} \operatorname{Ord}_{\Gamma}(f ; \zeta)
$$

where $\kappa(\Gamma ; \zeta)$ is the width of $\Gamma$ at $\zeta$ and $\operatorname{Ord}_{\Gamma}$ is the standard order function of $f$ at $\zeta$ with respect to $\Gamma$. This order does not depend on the group $\Gamma$ and is sometimes useful because for any $M=\left(\begin{array}{ll}\alpha & \beta \\ \gamma & \delta\end{array}\right) \in M_{2}^{+}(\mathbb{Z})$ and any $a / c \in \mathbb{Q}^{*}$ with $(a, c)=1$ we have a formula for the order of $f \mid M$ at $a / c$ :

$$
\operatorname{ord}\left(f \mid M ; \frac{a}{c}\right)=\frac{g^{2}}{m} \operatorname{ord}\left(f ; M\left(\frac{a}{c}\right)\right) \text {, }
$$

where $m=\operatorname{det} M$ and $g=(a \alpha+c \beta, a \gamma+c \delta)$. We can recognize $g$ as the factor which cancels when the fraction $M(a / c)=(a \alpha+c \beta) /(a \gamma+c \delta)$ is reduced to lowest terms. When $A \in \Gamma(1)$ and $A(\infty)=a / c$, this becomes

$$
\operatorname{ord}\left(f ; \frac{a}{c}\right)=\operatorname{ord}(f \mid A ; \infty)
$$

The function $\eta(\tau)$ is a modular form on $\Gamma(1)$ and for $A=\left(\begin{array}{ll}a & b \\ c & d\end{array}\right) \in \Gamma(1)$ its multiplier
is given by system is given by

$$
v_{\eta}(A)= \begin{cases}\left(\begin{array}{l}
d \\
c
\end{array}\right) e^{2 \pi i / 24\left|-3 c-a d\left(c^{2}-1\right)+c(a+d)\right|}, & c \text { odd } \\
\left(\begin{array}{l}
c \\
d
\end{array}\right)_{*} e^{2 \pi i / 24\left|3 d-3-b c\left(d^{2}-1\right)+d(b-c)\right|}, & \mathrm{d} \text { odd }\end{cases}
$$

The quantities $\left(\frac{d}{c}\right)$ and $\left(\frac{c}{d}\right)_{*}$ are equal to the Legendre-Jacobi symbol when the lower entry is positive and are extended to negative values of the lower entry in the following 
way: we set

and

$$
\operatorname{neg} x= \begin{cases}1, & x<0 \\ 0, & x \geq 0\end{cases}
$$

$$
\begin{gathered}
\left(\frac{d}{c}\right)=\left(\frac{d}{|c|}\right) \\
\left(\frac{c}{d}\right)_{*}=(-1)^{\operatorname{negc~neg} d}\left(\frac{c}{|d|}\right) .
\end{gathered}
$$

We shall need some properties of these extensions.

(i) Multiplicativity in the upper and lower entries separately.

(ii) Additivity in the upper entry:

$$
\left(\frac{d+c}{c}\right)=\left(\frac{d}{c}\right) \text { and }\left(\frac{c+k d}{d}\right)_{*}=(-1)^{\mathrm{ncg} d \mathrm{ncg} c(c+k d)}\left(\frac{c}{d}\right)_{*}
$$

(iii) Quadratic Reciprocity:

$$
\left(\frac{a}{b}\right)\left(\frac{b}{a}\right)=\left(\frac{a}{b}\right)_{*}\left(\frac{b}{a}\right)_{*}=(-1)^{\mathrm{ncg} a \mathrm{neg} b}(-1)^{(a-1)(b-1) / 4} .
$$

(iv) Additivity in the lower entry:

where

$$
\begin{aligned}
\left(\frac{a}{b+k a}\right)\left(\frac{a}{b}\right) & =\left(\frac{a}{b-k a}\right)_{*}\left(\frac{a}{b}\right)_{*} \\
& =(-1)^{\mathrm{ncg} a \operatorname{ncg} b(b+k a)} \epsilon(a, b, k),
\end{aligned}
$$

$$
\epsilon(a, b, k)= \begin{cases}+1, & \text { if } \equiv 0, a \equiv 1 \text { or } k \equiv 0(\bmod 4) \\ & \text { or if } a \equiv 2 \text { and } k \equiv b+2(\bmod 4) \\ -1, & \text { otherwise }\end{cases}
$$

We take (12) from Knopp [7] (but note that he chooses the argument by $-\pi<\arg z<\pi$ and so get $\left.(0 /-1)_{*}=-1\right)$ and refer the reader to Rankin [14] or Lehner [8] for general properties of modular forms and to Schoeneberg [15, ch. VI] for properties of $\Gamma_{0}(N)$. Equation (10) is from Biagioli [3] but a more accessible reference is Berndt [1, ch. 20 p. 333, lemma 0.1]. (Ligozat [9] has a related expression which has priority.)

3. The function $\boldsymbol{g}(\boldsymbol{\tau})$. We shall work in a slightly more general situation than that described in the introduction, with an eye to the applications made in Gordon and Sinor [6] and Gordon and Hughes [5].

Let $N \geq 1$ be an integer and let $r_{\delta}, s_{\delta} \in \mathbb{Z}$ for all positive $\sigma \mid N$ be such that

$$
k=\frac{1}{2} \sum_{\delta \mid N} r_{\delta}=\frac{1}{2} \sum_{\delta \mid N} s_{\delta}
$$


is an integer. We then define two eta-products

$$
\phi=\prod_{\delta \mid N}(\eta \circ \delta)^{r_{\delta}} \quad \text { and } \quad \psi=\prod_{\delta \mid N}(\eta \circ \delta)^{s_{\lambda}},
$$

and for any $d \mid N$ we let

$$
C(d)=\frac{N}{\left(N, d^{2}\right)} \sum_{\delta \mid N} \frac{(d, \delta)^{2}}{\delta} r_{\delta} \quad \text { and } \quad C^{\prime}(d)=\frac{N}{\left(N, d^{2}\right)} \sum_{\delta \mid N} \frac{(d, \delta)^{2}}{\delta} s_{\delta} .
$$

These are integers, and in addition to (17) we assume $r_{\delta}, s_{\delta}$ are such that

$$
(C(d), 24)=\left(C^{\prime}(d), 24\right) \text { for all } d \mid N
$$

and such that

$$
\prod_{\delta \mid N} \delta^{r_{\delta}-s_{\delta}} \text { is a rational square. }
$$

At a cusp $a / c \in \mathbb{Q}^{*}$ the group $\Gamma_{0}(N)$ has width $N /\left(N, d^{2}\right)$, where $d=(N, c)$, so (9), (10) and (11) give us

$$
\operatorname{Ord}_{\Gamma_{11}(N)}\left(\phi ; \frac{a}{c}\right)=\frac{C(d)}{24}, \quad \text { and } \quad \operatorname{Ord}_{\Gamma_{01}(N)}\left(\psi ; \frac{a}{c}\right)=\frac{C^{\prime}(d)}{24}
$$

If we express these fractions in lowest terms

$$
\frac{C(d)}{24}=\frac{c_{1 / d}}{e_{1 / d}} \text { and } \frac{C^{\prime}(d)}{24}=\frac{c_{1 / d}^{\prime}}{e_{1 / d}^{\prime}},
$$

then (18) shows that $e_{1 / d}=e_{1 / d}^{\prime}$, i.e., $\phi$ and $\psi$ have the same ramification indices at every cusp $a / c$. We also write $e$ and $e_{0}$ for $e_{1 / N}=e_{\infty}$ and $e_{1 / 1}$, respectively, and we can express $e_{1 / d}$ in terms of $C(d)$

$$
e_{1 / d}=\frac{24}{(24, C(d))}
$$

We define

$$
E=\text { l.c.m. }\left\{e_{1 / d}: d \mid N\right\}
$$

to be the least common multiple of all the ramification indices.

Suppose

$$
p \nmid N E \text { is prime }
$$

and define

$$
h=\frac{\phi \circ p}{\psi} .
$$

As noted by Gordon and Sinor [6], a modification of the criteria of Morris Newman [10] shows that $h$ is a function on $X_{0}\left(p N e_{0}, e\right)$, the Riemann surface of $\Gamma_{0}\left(p N e_{0}, e\right)$.

We choose $M, s$ and $t$ so that

$$
\begin{array}{ccc}
M>0, & N E \mid M, \quad p \nmid M \\
s, t>0, & e \mid t, \quad \text { and } & p s-M t=1 .
\end{array}
$$


We shall later impose additional conditions on $M, s$ and $t$ which shall need to be consistent with this choice. A complete set of right coset representatives for $\Gamma_{0}\left(N e_{0}, e\right)$ over $\Gamma_{0}\left(p N e_{0}, e\right)$ is $A_{0}, \ldots, A_{p}$, where

and

$$
A_{j}=\left(\begin{array}{cc}
1 & 0 \\
M j & 1
\end{array}\right) \text { for } j=0, \ldots, p-1,
$$

$$
A_{p}=\left(\begin{array}{cc}
s & t \\
M & p
\end{array}\right)
$$

We define a function $g$ to be the trace

$$
g=\sum_{j=0}^{p} h \circ A_{j}
$$

Then $g$ is a function on $X_{0}\left(N e_{0}, e\right)$.

In order to prove our theorem we shall write $g$ in terms of a Hecke operator and determine conditions under which $g$ is constant. Since $g$ has no poles in $\mathbb{t}$ it will be constant if and only if ord $(g ; \zeta) \geq 0$ for every cusp $\zeta \in \mathbb{Q}^{*}$.

4. Transferring $g$. We wish to get a lower bound for the order of $g$ at an arbitrary $\operatorname{cusp} \zeta=a / c \in \mathbb{Q}^{*}$.

Assuming that $(a, c)=1$, we set

$$
d=(N, c)
$$

and we choose $y, z$ so that

$$
A=\left(\begin{array}{ll}
a & y \\
c & z
\end{array}\right) \in \Gamma(1)
$$

Since $p \nmid N E$ and $\Gamma_{0}\left(N e_{0}, e\right) \geq \Gamma(N E)$, then for the purpose of computing the order of $g$ we may replace $\zeta$, if necessary, by a cusp equivalent to it modulo $N$ so as to guarantee

$$
a, c, y, z \geq 0 \text { and } p \mid a .
$$

The conditions (26) and similar conditions we shall impose in the future are only for convenience in the analysis and are not inherent restrictions, i.e. one should be able to carry through the analysis in their absence but there may be some additional difficulties.

We define

$$
g^{*}=g \circ A=g \mid A .
$$

Then by $(11), \operatorname{ord}(g ; a / c)=\operatorname{ord}\left(g^{*} ; \infty\right)$. In more detail, $g^{*}$ is given by

$$
g^{*}=\sum_{j=0}^{p} \prod_{\delta \mid N} \frac{\left(\eta \circ p \delta \circ A_{j} A\right)^{r_{\delta}}}{\left(\eta^{\circ} \delta \circ A_{j} A\right)^{s_{\triangleright}}} .
$$

We examine this function piecemeal, first looking at one factor of one term. 
Fix a divisor $\delta$ of $N$ and set

$$
\delta^{\prime}=(\delta, c)=(\delta, d), \quad c^{\prime \prime}=\frac{c}{\delta^{\prime}}, \quad \delta^{\prime \prime}=\frac{\delta}{\delta^{\prime}},
$$

and

$$
\gamma=\frac{c+a M j}{\delta}
$$

When $j<p$ the product of matrices in the denominator of $(28)$ is

$$
\begin{aligned}
\left(\begin{array}{ll}
\delta & 0 \\
0 & 1
\end{array}\right) A_{j} A & =\left(\begin{array}{cc}
\delta a & \delta y \\
c+a M j & z+y M j
\end{array}\right) \\
& =\left(\begin{array}{cc}
\delta^{\prime \prime} a & \delta y \\
\gamma & z+y M j
\end{array}\right)\left(\begin{array}{ll}
\delta^{\prime} & 0 \\
0 & 1
\end{array}\right) \\
& =\left(\begin{array}{cc}
\delta^{\prime \prime} a & \delta y \\
\gamma & z+y M j
\end{array}\right)\left(\begin{array}{cc}
1 & b / \delta^{\prime \prime} \\
0 & 1 / \delta^{\prime \prime}
\end{array}\right)\left(\begin{array}{cc}
\delta^{\prime} & -b \\
0 & \delta^{\prime \prime}
\end{array}\right) \\
& =\left(\begin{array}{cc}
\delta^{\prime \prime} a & b a+\delta^{\prime} y \\
\gamma & (z+y M j+b \gamma) / \delta^{\prime \prime}
\end{array}\right)\left(\begin{array}{cc}
\delta^{\prime} & -b \\
0 & \delta^{\prime \prime}
\end{array}\right)
\end{aligned}
$$

All the entries of these matrices will be integers if we choose $b \in \mathbb{Z}$ so that

$$
\begin{gathered}
-b c^{\prime \prime} \equiv z\left(\bmod \delta^{\prime \prime}\right), \\
b>0 \quad \text { if } \delta^{\prime \prime}>1, \quad \text { and } \\
b=0 \quad \text { if } \delta^{\prime \prime}=1,
\end{gathered}
$$

which is possible because $\left(c^{\prime \prime}, \delta^{\prime \prime}\right)=1$. Thus we have

$$
\left(\begin{array}{ll}
\delta & 0 \\
0 & 1
\end{array}\right) A_{j} A=B\left(\begin{array}{cc}
\delta^{\prime} & -b \\
0 & \delta^{\prime \prime}
\end{array}\right)
$$

where $B=\left(\begin{array}{ll}\alpha & \beta \\ \gamma & \xi\end{array}\right) \in \Gamma(1)$ has entries

$$
\begin{cases}\alpha=\delta^{\prime \prime} a & \beta=b a+\delta^{\prime} y, \\ \gamma=(c+a M j) / \delta^{\prime}, & \zeta=(z+y M j+b \gamma) / \delta^{\prime \prime} .\end{cases}
$$

Since $b \geq 0$ all of $\alpha, \beta, \gamma$, and $\zeta$ are non-negative.

Still working in the case $j<p$ we consider the product of matrices in the numerator. Choose $b_{1} \geq 0$ so that

$$
-b_{1} \gamma \equiv z+y M j\left(\bmod p \delta^{\prime \prime}\right)
$$

and let

$$
\beta_{1}=b_{1} a+\delta^{\prime} y, \quad \zeta_{1}=\left(z+y M j+b_{1} \gamma\right) / \delta^{\prime \prime} .
$$

Then $B_{1}=\left(\begin{array}{cc}p \alpha & \beta_{1} \\ \gamma & \zeta_{1} / p\end{array}\right) \in \Gamma(1)$ satisfies

$$
\left(\begin{array}{cc}
p \delta & 0 \\
0 & 1
\end{array}\right) A_{j} A=B_{1}\left(\begin{array}{cc}
\delta^{\prime} & -b_{1} \\
0 & p \delta^{\prime \prime}
\end{array}\right)
$$


In fact, we want to be more particular about our choice of $b_{1}$ : First choose $L$ to be a multiple of $M$ satisfying

$$
-L c \equiv z+y M j \quad(\bmod p)
$$

which is possible because $p \mid a$ and so $p \nmid c$, and then we set

$$
b_{1}=L \delta^{\prime}+p s b \text {. }
$$

What we want to note about this choice is that $b$ depends on $\delta$, but not on $j$, and that $L$ depends on $j$ but not on $\delta$, so we have separated the effect of the two parameters.

When $j=p$ we have

$$
\left(\begin{array}{ll}
\delta & 0 \\
0 & 1
\end{array}\right) A_{p} A=B\left(\begin{array}{cc}
\delta^{\prime} & -b \\
0 & \delta^{\prime \prime}
\end{array}\right)
$$

and

$$
\left(\begin{array}{cc}
p \delta & 0 \\
0 & 1
\end{array}\right) A_{p} A=B_{1}\left(\begin{array}{cc}
p \delta^{\prime} & -p b \\
0 & \delta^{\prime \prime}
\end{array}\right)
$$

where now $B=\left(\begin{array}{ll}\alpha & \beta \\ \gamma & \zeta\end{array}\right)$ and $B_{1}=\left(\begin{array}{cc}\alpha & p \beta \\ \gamma / p & \zeta\end{array}\right)$ are both in $\Gamma(1)$ with $\alpha, \beta, \gamma$ and $\zeta$ defined by

$$
\begin{cases}\alpha=\delta^{\prime \prime}(s a+t c), & \beta=b(s a+t c)+\delta^{\prime}(s y+t z) \\ \gamma=(M a+p c) / \delta^{\prime}, & \xi=(M y+p z+b \gamma) / \delta^{\prime \prime}\end{cases}
$$

Applying the transformation property of $\eta$ we get, first for $j<p$,

and

$$
\eta \circ p \delta \circ A_{j} A=v_{\eta}\left(B_{1}\right) \sqrt{\frac{(c+a M j) \tau+(z+y M j)}{p}} \eta \circ\left(\begin{array}{cc}
\delta^{\prime} & -b_{1} \\
0 & p \delta^{\prime \prime}
\end{array}\right),
$$

$$
\eta \circ \delta \circ A_{j} A=v_{\eta}(B) \sqrt{(c+a M j) \tau+(z+y M j)} \eta \circ\left(\begin{array}{cc}
\delta^{\prime} & -b \\
0 & \delta^{\prime \prime}
\end{array}\right)
$$

Since

$$
\left(\begin{array}{cc}
\delta^{\prime} & -b_{1} \\
0 & p \delta^{\prime \prime}
\end{array}\right)=\left(\begin{array}{cc}
\delta^{\prime} & -b s \\
0 & \delta^{\prime \prime}
\end{array}\right)\left(\begin{array}{cc}
1 & -L \\
0 & p
\end{array}\right)
$$

this gives us

$$
\frac{\left(\eta \circ p \delta \circ A_{j} A\right)^{r_{o}}}{\left(\eta \circ \delta \circ A_{j} A\right)^{s_{\delta}}}=\xi_{\delta, j} p^{-k} \times \frac{\left(\eta \circ\left(\begin{array}{cc}
\delta^{\prime} & -b s \\
0 & \delta^{\prime \prime}
\end{array}\right)\right)^{r^{\prime}} \circ\left(\begin{array}{cc}
1 & -L \\
0 & p
\end{array}\right)}{\left(\eta \circ\left(\begin{array}{cc}
\delta^{\prime} & -b \\
0 & \delta^{\prime \prime}
\end{array}\right)\right)^{s_{\delta}}}
$$

where $\xi_{\delta, j}$ is the constant

$$
\xi_{\delta, j}=\frac{v_{\eta}\left(B_{1}\right)^{r_{\partial}}}{v_{\eta}(B)^{s_{\phi}}}
$$


and when $j=p$,

$$
\frac{\left(\eta \circ p \delta \circ A_{p} A\right)^{r_{\nu}}}{\left(\eta \circ \delta \circ A_{p} A\right)^{s_{\diamond}}}=\xi_{\delta, j} \times \frac{\left(\eta \circ\left(\begin{array}{cc}
\delta^{\prime} & -p b \\
0 & \delta^{\prime \prime}
\end{array}\right)\right)^{r_{\diamond}} \circ\left(\begin{array}{ll}
p & 0 \\
0 & 1
\end{array}\right)}{\left(\eta \circ\left(\begin{array}{cc}
\delta^{\prime} & -b \\
0 & \delta^{\prime \prime}
\end{array}\right)\right)^{s_{\lambda}}}
$$

where $\xi_{\delta, p}$ is still as in (38), but $B$ and $B_{1}$ are defined by (37). Note the absence in the second expression of the factor $p^{-k}$.

Consequently we now set

$$
\begin{aligned}
\phi^{*} & =\prod_{\delta \mid N}\left(\eta \circ\left(\begin{array}{cc}
\delta^{\prime} & -b s \\
0 & \delta^{\prime \prime}
\end{array}\right)\right)^{r_{\phi}}, \\
\phi^{* *} & =\prod_{\delta \mid N}\left(\eta \circ\left(\begin{array}{cc}
\delta^{\prime} & -p b \\
0 & \delta^{\prime \prime}
\end{array}\right)\right)^{r_{\lambda}},
\end{aligned}
$$

and

$$
\psi^{*}=\prod_{\delta \mid N}\left(\eta \circ\left(\begin{array}{cc}
\delta^{\prime} & -b \\
0 & \delta^{\prime \prime}
\end{array}\right)\right)^{s_{d}}
$$

and we let

$$
\xi_{j}=\prod_{\delta \mid N} \xi_{\delta, j}
$$

Then we have

$$
g^{*}=\left(\sum_{j=0}^{n-1} \xi_{j} p^{-k} \phi^{*} \circ\left(\begin{array}{ll}
1 & L \\
0 & p
\end{array}\right)+\xi_{p} \phi^{* *} \circ p\right) / \psi^{*}
$$

5. Coincidence of the coefficients. We now show that $\xi_{j}=\xi_{0}$ for $j<p$ and we determine the ratio $\epsilon_{d}(p)=\xi_{p} / \xi_{0}$. We note that when $j=0$ the values of $\alpha, \beta, \gamma$ and $\zeta$ are

$$
\begin{cases}\alpha_{0}=\delta^{\prime \prime} a, & \beta_{0}=a b+\delta^{\prime} y, \\ \gamma_{0}=c^{\prime \prime}, & \zeta_{0}=\left(z-b \gamma_{0}\right) / \delta^{\prime \prime} .\end{cases}
$$

We first consider a prime $p>3$; the details are different when $p=2$ or $p=3$. Here we impose the additional conditions on $M$ and $t$ that

$$
M \equiv t \equiv 0 \quad(\bmod 24),
$$

and $b$ is such that $\zeta$ is odd. These are consistent with the previous conditions (22)-(31). We can write

where

$$
\xi_{\delta, j}=L_{\delta, j} \exp \left(2 \pi i / 24 . E_{\delta, j}\right)
$$

and

$$
L_{\delta, j}=\left(\frac{\gamma}{\xi_{1} / p}\right)_{*}^{r_{\lambda}} /\left(\frac{\gamma}{\xi}\right)_{*}^{s_{\delta}}
$$

$E_{\delta, j}=\left[3 \zeta_{1} / p-3-\beta_{1} \gamma\left(\zeta_{1}^{2} / p^{2}-1\right)+\zeta_{1} / p\left(\beta_{1}-\gamma\right)\right] r_{\delta}-\left[3 \zeta-3-\beta \gamma\left(\zeta^{2}-1\right)+\zeta(\beta-\gamma)\right] s_{\delta}$. 
The congruences (43) allow us to derive the following congruences modulo 24:

$$
b_{1} \equiv b, \quad \beta_{1} \equiv \beta \equiv \beta_{0}, \quad \zeta_{1} \equiv \zeta \equiv \zeta_{0}, \quad \gamma \equiv \gamma_{0} .
$$

Keeing in mind that the entries of $B$ and $B_{1}$ are non-negative, we have

and

$$
\left(\frac{\gamma}{p}\right)=\left(\frac{\delta^{\prime}}{p}\right)\left(\frac{c+a M j}{p}\right)
$$

$$
\left(\frac{\gamma}{\zeta_{1}}\right)=\left(\frac{\gamma}{\zeta}\right)=\left(\frac{-\beta}{\zeta}\right)=\left(\frac{-\beta}{\zeta_{0}}\right)=\left(\frac{\gamma_{0}}{\zeta_{0}}\right)
$$

because $4 \gamma \mid \zeta_{1}-\zeta$ and $4 \beta \mid \zeta-\zeta_{0}$. Now setting $L_{j}=\prod_{\delta \mid N} L_{\delta, j}$, we have

$$
L_{j}=\prod_{\delta \mid N}\left(\frac{\delta^{\prime}}{p}\right)^{r_{\delta}}\left(\frac{\gamma_{0}}{\zeta_{0}}\right)^{r_{j}-s_{\lambda}}=L_{0}
$$

We also set $E_{j}=\sum_{\delta \mid N} E_{\delta, j}$ and the congruences (45) show that, modulo 24,

$$
E_{j} \equiv E_{0} \equiv \sum_{\delta \mid N}\left(r_{\delta}-s_{\delta}\right)\left[-3-\beta_{0} \gamma_{0}\left(\zeta_{0}^{2}-1\right)\right]+\sum_{\delta \mid N}\left(p r_{\delta}-s_{\delta}\right)\left[\zeta_{0}\left(3+\beta_{0}-\gamma_{0}\right)\right] .
$$

Together (46) and (47) give us

$$
\xi_{j}=\xi_{0} \text { for all } j<p .
$$

When $j=p$ we use (37) to derive some helpful relations:

$$
\left\{\begin{array}{l}
\zeta=p \zeta_{0}+(M / \delta) \beta_{0}, \quad s \zeta=\zeta_{0}+(M / \delta) \beta_{0}, \\
\beta=s \beta_{0}+\delta^{\prime} \zeta_{0} t
\end{array}\right.
$$

and we have the following congruences modulo 24

$$
\alpha \equiv p \alpha_{0}, \quad \beta \equiv p \beta_{0}, \quad \gamma \equiv p \gamma_{0}, \quad \zeta \equiv p \zeta_{0} .
$$

$\zeta$ is odd, so we take

$$
L_{\delta, p}=\left(\frac{\gamma / p}{\zeta}\right)^{r_{\lambda}} /\left(\frac{\gamma}{\zeta}\right)^{s_{n}}
$$

and

$$
E_{\delta, p}=\left[3 \zeta-3-\beta \gamma\left(\zeta^{2}-1\right)+\zeta(p \beta-\gamma / p)\right] r_{\delta}-\left[3 \zeta-3-\beta \gamma\left(\zeta^{2}-1\right)+\zeta(\beta-\gamma)\right] s_{\delta},
$$

and let $L_{p}=\prod_{\delta \mid N} L_{\delta, p}$ and $E_{p}=\sum_{\delta \mid N} E_{\delta, p}$. Now we have

$$
\left(\frac{p}{\zeta}\right)=(-1)^{(p-1)(\zeta-1) / 4}\left(\frac{M \delta \beta_{0}}{p}\right)=\left(\frac{M y}{p}\right)\left(\frac{\delta^{\prime \prime}}{p}\right) \exp \left(\frac{\pi i}{4}(p-1)\left(p \zeta_{0}-1\right)\right),
$$

and

$$
\begin{aligned}
\left(\frac{\gamma}{\zeta}\right) & =\left(\frac{-\beta}{\zeta}\right)=\left(\frac{-\beta}{s}\right)\left(\frac{-\beta}{\zeta_{0}}\right)=\left(\frac{-\delta^{\prime} t}{s}\right)\left(\frac{\zeta_{0}}{s}\right)\left(\frac{s}{\zeta_{0}}\right)\left(\frac{-\beta_{0}}{\zeta_{0}}\right) \\
& =\left(\frac{\delta^{\prime} t}{p}\right)\left(\frac{\gamma_{0}}{\zeta_{0}}\right) \exp \left(\frac{\pi i}{4}(p-1)\left(\zeta_{0}-1\right)\right) .
\end{aligned}
$$


Hence

$$
L_{p}=L_{0}\left[\prod_{\delta \mid N}\left(\frac{\delta^{\prime \prime}}{p}\right)^{r_{\delta}}\left(\frac{\delta^{\prime}}{p}\right)^{s_{\delta}}\right] \exp \left(\frac{\pi i}{4}(p-1) \sum_{\delta \mid N} s_{\delta}\left(\zeta_{0}-1\right)\right) .
$$

Using the congruences (49) we get

Hence

$$
E_{p} \equiv E_{0}-3(p-1) \sum_{\delta \mid N} s_{\delta} \zeta_{0} \quad(\bmod 24) .
$$

$$
\xi_{p}=L_{p} e^{2 \pi i / 24 \cdot E_{p}}=\xi_{0} \cdot \epsilon_{d}(p),
$$

where

$$
\epsilon_{d l}(p)=\left[\prod_{\delta \mid N}\left(\frac{\delta^{\prime \prime}}{p}\right)^{r_{s}}\left(\frac{\delta^{\prime}}{p}\right)^{s_{s}}\right]\left(\frac{(-1)^{k}}{p}\right) .
$$

This proves our claim when $p>3$. When $p=3$ we impose a different set of conditions than (43), namely

$$
\left\{\begin{array}{l}
\text { (i) } M \equiv t \equiv 0(\bmod 8), \\
\text { (ii) Choose } b \text { so that } \zeta_{0} \text { is odd, } \\
\text { (iii) } 9|a, 9| z, 3|b, 3| s, \\
\text { (iv) } L c \equiv-2 y M j(\bmod 9) .
\end{array}\right.
$$

The conditions (54i, ii) allow us to derive (46) and (50) exactly as before. However, following the previous proofs of the congruences (47) and (51), we find that they are only correct if we consider the congruences as being modulo 8 , rather than modulo 24 .

So we must consider the modulus 3 separately. For $j<p=3$ we find that

$$
\zeta_{1} / 3 \equiv \zeta \equiv y M j \delta^{\prime \prime}, \quad \beta \gamma \equiv-1, \quad \text { and } \quad \beta_{1} \equiv \beta \equiv-\gamma \equiv-c \delta^{\prime},
$$

so that

$$
E_{\delta, j} \equiv\left(r_{\delta}-s_{\delta}\right)\left(j^{2}-1\right)-M_{j}\left(r_{\delta}-s_{\delta}\right) \delta .
$$

Since $p=3$ does not divide the ramification index $e=e_{1 / N}$ we must have $C(N) \equiv C^{\prime}(N) \equiv$ $0(\bmod 3)$, and this says

$$
\sum_{\delta \mid N} \delta r_{\delta} \equiv \sum_{\delta \mid N} \delta s_{\delta} \equiv 0 \quad(\bmod 3)
$$

which reduces $(55)$ to

$$
E_{j}=\sum_{\delta \mid N} E_{\delta, j} \equiv 0 \quad(\bmod 3),
$$

and so (47) is still true modulo 24 .

For $j=p=3$ we use (54ii-iv) to get

and so

$$
E_{\delta, p} \equiv r_{\delta}[-M c y \delta] \quad(\bmod 3)
$$

$$
E_{p} \equiv 0 \equiv E_{0} \quad(\bmod 3) \text {. }
$$


Now we consider $p=2$. The conditions we impose in place of (43) are

$$
\left\{\begin{array}{l}
\text { (i) } M \equiv t \equiv y z \equiv a c \equiv 0(\bmod 3) \\
\text { (ii) } M \equiv s \equiv-c \equiv y \equiv 1(\bmod 8) \\
\text { (iii) } 16|a, 16| z, 8 \mid b, \\
\text { (iv) } L c \equiv-y M j(\bmod 16) .
\end{array}\right.
$$

For $j<p, \gamma$ is odd, so we must consider

$$
L_{\delta, j}=\left(\frac{\zeta_{1} / 2}{\gamma}\right)^{r_{\lambda}} /\left(\frac{\zeta}{\gamma}\right)^{s_{\diamond}} \text {. }
$$

Since $8 \mid a$ we get

$$
\left(\frac{\zeta_{1}}{\gamma}\right)=\left(\frac{\zeta}{\gamma}\right)=\left(\frac{\alpha}{\gamma}\right)=\left(\frac{\alpha_{0}}{\gamma_{0}}\right)=\left(\frac{\zeta_{0}}{\gamma_{0}}\right),
$$

and from $8 \mid a$ and $8 \mid c+1$ we get

$$
\left(\frac{2}{\gamma}\right)=\left(\frac{2}{\delta^{\prime}}\right)
$$

so that $L_{j}=\prod_{\delta \mid N} L_{\delta, j}$ is

$$
L_{j}=L_{0}=\prod_{\delta \mid N}\left(\frac{2}{\delta^{\prime}}\right)^{r_{0}}\left(\frac{\zeta_{0}}{\gamma_{0}}\right)^{r_{D}-s_{o}}
$$

Next let

$$
E_{\delta, j}=r_{\delta}\left[-3 \gamma-\alpha \zeta_{1}\left(\gamma^{2}-1\right)+\gamma(2 \alpha+\zeta / 2)\right]-s_{\delta}\left[-3 \gamma-\alpha \zeta\left(\gamma^{2}-1\right)+\gamma(\alpha+\zeta)\right]
$$

and $E_{j}=\sum_{\delta \mid N} E_{\delta, j}$. We again find a congruence modulo 24 by doing the moduli 3 and 8 separately.

Modulo 3 the congruence $M \equiv 0$ leads to

$$
E_{\delta, j} \equiv\left(s_{\delta}-r\right) \delta\left[-3 \gamma_{0}-\alpha_{0} \zeta_{0}\left(\gamma_{0}^{2}-1\right)\right]+\left(2 s_{\delta}-r_{\delta}\right)\left[\gamma_{0}\left(\alpha_{0}+\zeta_{0}\right)\right] .
$$

Modulo 8 the conditions (56) imply that

$$
b_{1} \equiv L \delta^{\prime}, \quad \gamma^{2} \equiv 1, \quad \gamma \equiv \gamma_{0}, \quad \alpha \equiv 0 \quad \text { and } \quad \zeta / w \equiv \zeta \equiv y M \delta^{\prime \prime} j .
$$

Hence we get

$$
E_{\delta, j} \equiv\left(s_{\delta}-r_{\delta}\right)\left[-3 \gamma_{0}+\delta_{j}\right]
$$

Since $p=2 \nmid E$ we must have $C(N) \equiv C^{\prime}(N) \equiv 0(\bmod 8)$, or $\sum \delta r_{\delta} \equiv \sum \delta s_{\delta} \equiv 0(\bmod 8)$, and so

$$
E_{j} \equiv \sum_{\delta \mid N}\left(-3 \gamma_{0}\right)\left(s_{\delta}-r_{\delta}\right)(\bmod 8)
$$

Since no quantity in $(57),(58)$ or $(59)$ depends on $j$ we see that $\xi_{1}=\xi_{0}$. 
When $j=p=2$, then $\gamma$ is even (see (37)), and we consider

and

$$
L_{\delta, p}=\left(\frac{\gamma / 2}{\zeta}\right)^{r_{s}} /\left(\frac{\gamma}{\zeta}\right)^{s_{n}}
$$

$$
E_{\delta, p}=r_{\delta}\left[3 \zeta-3-\beta \gamma\left(\zeta^{2}-1\right)+\zeta(2 \beta-\gamma / 2)\right]-s_{\delta}\left[3 \zeta-3-\beta \gamma\left(\zeta^{2}-1\right)+\zeta(\beta-\gamma)\right] .
$$

Since $8 \mid \zeta_{0}$, the relations (48) and the congruences (56) show that

$$
\left(\frac{2}{\zeta}\right)=\left(\frac{2}{M \delta \beta_{0}}\right)=\left(\frac{2}{\delta \delta^{\prime}}\right)=\left(\frac{2}{\delta^{\prime \prime}}\right) \text {. }
$$

We also use these relations and congruences to analyze $(\gamma / \zeta)$ : We have

and

$$
\left(\frac{\gamma}{\zeta}\right)=\left(\frac{-\beta}{\zeta}\right)=(-1)^{\left(\delta^{\prime}+1\right) / 2 \cdot\left(\delta^{\prime \prime}-1\right) / 2}\left(\frac{\zeta}{-\beta}\right)
$$

$$
\begin{aligned}
\left(\frac{\zeta}{-\beta}\right) & =\left(\frac{s}{-\beta}\right)\left(\frac{\zeta_{0}}{-\beta}\right)=\left(\frac{-\beta}{s}\right)\left(\frac{\zeta_{0}}{-s \beta_{0}}\right)=\left(\frac{-\delta^{\prime} \zeta_{0} t}{s}\right)\left(\frac{\zeta_{0}}{-s \beta_{0}}\right) \\
& =\left(\frac{s}{\delta^{\prime} t}\right)\left(\frac{\zeta_{0}}{\gamma_{0}}\right)=\left(\frac{2}{\delta^{\prime} t}\right)\left(\frac{\zeta_{0}}{\gamma_{0}}\right)=\left(\frac{2}{\delta^{\prime}}\right)\left(\frac{\zeta_{0}}{\gamma_{0}}\right) .
\end{aligned}
$$

Therefore $L_{p}=\prod_{\delta} L_{\delta, p}$ satisfies

$$
L_{p}=L_{0} \prod_{\delta \mid N}\left(\frac{2}{\delta^{\prime \prime}}\right)^{r_{0}}\left(\frac{2}{\delta^{\prime}}\right)^{r_{\nu}} \exp \left\{(\pi i / 4) \sum_{\delta \mid N}\left(r_{\delta}-s_{\delta}\right)\left(\delta^{\prime}+1\right) / 2 .\left(\delta^{\prime \prime}-1\right) / 2\right\} .
$$

As before, we consider the congruences for $E_{\delta, p}$ modulo 3 and modulo 8 separately. Modulo 3 we have

so

$$
s \equiv p, \quad \alpha \equiv p \alpha_{0}, \quad \beta \equiv p \beta_{0}, \quad \gamma \equiv p \gamma_{0}, \quad \text { and } \quad \zeta \equiv p \zeta_{0},
$$

$$
E_{\delta, p} \equiv\left(s_{\delta}-r_{\delta}\right)\left[-\beta_{0} \gamma_{0}\left(\zeta_{0}^{2}-1\right)\right]+\left(2 r_{\delta}-s_{\delta}\right)\left[\zeta_{0}\left(\beta_{0}-\gamma_{0}\right)\right]
$$

Modulo 8 we use the relations (48) and the congruences (56) to get

$$
E_{\delta, 2} \equiv r_{\delta}\left[3 \delta^{\prime \prime}-3+\delta\right]-s_{\delta}\left[3 \delta^{\prime \prime}-3\right]
$$

and so $E_{2}=\sum_{\delta} E_{\delta, 2}$ satisfies

$$
E_{2} \equiv \sum_{\delta \mid N}\left(r_{\delta}-s_{\delta}\right) \cdot 3 \delta^{\prime \prime} \equiv E_{0}+3 \sum_{\delta \mid N}\left(r_{\delta}-s_{\delta}\right)\left(\delta^{\prime \prime}-\delta^{\prime}\right) \quad(\bmod 8)
$$

Combining the congruences for $E_{2}$ and $E_{0}$ modulo 3 we see that

$$
E_{2} \equiv E_{0}+\sum_{\delta \mid N} r_{\delta}\left(\gamma_{0} \zeta_{0}+1\right)\left(\alpha_{0} \gamma_{0}-\beta_{0} \zeta_{0}-1\right)+\sum_{\delta \mid N} s_{\delta}\left(\gamma_{0} \zeta_{0}-1\right)\left(\beta_{0} \zeta_{0}-\alpha_{0} \gamma_{0}-1\right) \quad(\bmod 3) \text {. }
$$

The sums on the right are congruent to $0(\bmod 3)$ whenever $3 \nmid \gamma_{0}$ and $3 \nmid \zeta_{0}$. When $3 \mid \gamma_{0}$ 
then we impose the condition $3 \mid b$ in addition to the conditions (56). Then we have

and

$$
\sum_{\delta \mid N} r_{\delta}\left(-\beta_{0} \zeta_{0}-1\right) \equiv \sum_{\delta \mid N} r_{\delta}(\delta y z-1) \equiv-2 k \quad(\bmod 3)
$$

$$
\sum_{\delta \mid N} s_{\delta}\left(-\beta_{0} \zeta_{0}+1\right) \equiv 2 k \quad(\bmod 3)
$$

This, and a similar reasoning when $3 \mid \zeta_{0}$, shows that

$$
E_{2} \equiv E_{0} \quad(\bmod 3)
$$

in all cases.

Thus, modulo 24 we now have

$$
E_{2} \equiv E_{0}+3 \sum_{\delta \mid N}\left(r_{\delta}-s_{\delta}\right)\left(\delta^{\prime \prime}-\delta^{\prime}\right) \quad(\bmod 24),
$$

which combines with $(60)$ to give us

$$
\begin{aligned}
\xi_{2} & =\xi_{0} \cdot \prod_{\delta \mid N}\left(\frac{2}{\delta^{\prime \prime}}\right)^{r_{\lambda}}\left(\frac{2}{\delta^{\prime}}\right)^{s_{\phi}}\left(\frac{(-1)^{r_{0}-s_{\phi}}}{\delta}\right) \\
& =\xi_{0} \cdot \prod_{\delta \mid N}\left(\frac{2}{\delta^{\prime \prime}}\right)^{r_{\delta}}\left(\frac{2}{\delta^{\prime}}\right)^{s_{n}},
\end{aligned}
$$

because $\sum_{\delta}(\delta-1) / 2 .\left(r_{\delta}-s_{\delta}\right) \equiv 0(\bmod 2)$

Conclusion of the proof. The previous section showed that $\xi_{j}=\xi_{0}$ when $j<p$ and that $\xi_{p}=\xi_{0} . \epsilon_{d}(p)$, where

$$
\epsilon_{d}(p)= \begin{cases}\left(\frac{(-1)^{k}}{p}\right) \cdot \prod_{\delta \mid N}\left(\frac{\delta^{\prime \prime}}{p}\right)^{r_{\lambda}}\left(\frac{\delta^{\prime}}{p}\right)^{s_{\diamond}}, & p>2, \\ \prod_{\delta \mid N}\left(\frac{2}{\delta^{\prime \prime}}\right)^{r_{\delta}}\left(\frac{2}{\delta^{\prime}}\right)^{s_{\delta}}, & p=2 .\end{cases}
$$

Hence (41) can be rewritten as

$$
g^{*}=p^{k-1} \xi_{0}\left(\frac{1}{p} \sum_{j=0}^{p-1} \phi^{*} \circ\left(\begin{array}{ll}
1 & L \\
0 & p
\end{array}\right)+p^{k-1} \epsilon_{d}(p) \phi^{* *} \circ p\right) / \psi^{*}
$$

We set $L=e_{1 / d} l$, and let $G^{*}=g^{*} \circ e_{1 / d}, \Phi^{*}=\phi^{*} \circ e_{1 / d}, \Phi^{* *}=\phi^{* *} \circ e_{1 / d}$, and $\Phi^{*}=\psi^{*} \circ e_{1 / d}$, and rewrite this as

$$
G^{*}=p^{k-1} \xi_{0}\left(\frac{1}{p} \sum_{j=0}^{p-1} \Phi^{*} \circ\left(\begin{array}{ll}
1 & l \\
0 & p
\end{array}\right)+p^{k-1} \epsilon_{d}(p) \Phi^{* *} \circ p\right) / \Psi^{*}
$$

Since the width of $\Gamma_{0}(N)$ at $a / c$ is $N /\left(N, d^{2}\right)$, we express the functions occurring here in terms of $X=\exp \left(2 \pi i \tau\left(N, d^{2}\right) / N\right)$. From (39) we see that

$$
\phi^{*}=X^{C(d) / 24} \sum_{n=0}^{\infty} b_{n} X^{n}
$$


and consequently

$$
\Phi^{*}=\sum_{m \geq c_{3 / d}} a_{m} X^{m}
$$

where $a_{m}=0$ except when $n \equiv c_{1 / d}\left(\bmod e_{1 / d}\right)$. In particular, $a_{c_{1 / d}}=b_{0} \neq 0$. The same is true for $\Phi^{* *}$, and it will also be true for $\Psi^{*}$ if we replace $c_{1 / d}$ by $c_{1 / d}^{\prime}$.

Since $p \nmid L$ and $L$ is a multiple of $N E$, then $l$ is a multiple of $N$. Hence

$$
\frac{1}{p} \sum_{j=0}^{p-1} \Phi^{*} \circ\left(\begin{array}{ll}
1 & l \\
0 & p
\end{array}\right)=\sum_{p m \geq c_{1 / d}} a_{p m} X^{m}=\Phi^{*} \mid U_{p} .
$$

(Note that this last equation is an extension of the definition (3) of the operator $\mid U_{p}$.) We also set $\Phi^{* *} \mid V_{p}=p^{k-1} \Phi^{*} \circ p$ and (62) becomes

$$
G^{*}=p^{1-k} \xi_{0}\left(\Phi^{*}\left|U_{p}+\epsilon_{d}(p) \Phi^{* *}\right| V_{p}\right) / \Psi^{*} .
$$

We let $m_{0}$ be the order of $G^{*}$ in terms of the variable $X$, so that $m_{0}=$ $N /\left(N, d^{2}\right) \operatorname{ord}\left(G^{*} ; \infty\right)$, and work out a lower bound for $m_{0}$. This will give us a lower bound on the order of $g$ at $a / c$ because

$$
\begin{aligned}
\operatorname{Ord}_{\Gamma_{1 /}(N)}\left(g ; \frac{a}{c}\right) & =\frac{N}{\left(N, d^{2}\right)} \operatorname{ord}\left(g^{*} ; \infty\right) \\
& =\frac{N}{\left(N, d^{2}\right)} \frac{1}{e_{1 / d}} \operatorname{ord}\left(G^{*} ; \infty\right) \\
& =\frac{m_{0}}{e_{1 / d}} .
\end{aligned}
$$

In terms of $X, \Psi^{*}$ has order $c_{1 / d}^{\prime}$ and $\Phi^{* *} \mid V_{p}$ has order $p c_{1 / d}$. The order of $\Phi^{*} \mid U_{p}$ is the least integer $m$ such that $c_{1 / d} \leq p m$ and $a_{p m} \neq 0$. The last condition necessitates that $p m \equiv c_{1 / d}\left(\bmod e_{1 / d}\right)$, which is equivalent to $m \equiv p c_{1 / d}\left(\bmod e_{1 / d}\right)$, since $p \nmid e_{1 / d}$ and $e_{1 / d}$ is a factor of 24.

If $C(d)<0$ then $m>c_{1 / d}>p c_{1 / d}$, so $m_{0} \geq p c_{1 / d}-c_{1 / d}^{\prime}$.

If $C(d)>0$ we choose $j$ to be the least positive integer such that $j \equiv p c_{1 / d}\left(\bmod e_{1 / d}\right)$. Then $j \leq m$ and $j \leq p c_{1 / d}$, so both $\Phi^{*} \mid U_{p}$ and $\Phi^{* *} \mid V_{p}$ have order at least $j$. Hence $m_{0} \geq j-c_{1 / d}^{\prime}$.

If $C(d)=0$ then $m_{0} \geqslant-c_{1 / d}^{\prime}$.

We now specialize to the situation of the introduction. We take $\phi=\psi=f$, i.e., we are taking $r_{\delta}=s_{\delta}$ for all $\delta$, we assume that $p \equiv 1(\bmod E)$, and we assume that $0 \leq C(d) \leq 24$. Then $j=c_{1 / d}=c_{1 / d}^{\prime}$ so our lower bounds on the order of $G^{*}$ now show that

$$
\operatorname{Ord}_{\Gamma_{10}(N)}\left(g ; \frac{a}{c}\right) \geqslant 0
$$

for all cusps $a / c \in \mathbb{Q}^{*}$. Since $g$ is a modular form of degree 0 on $\Gamma_{0}(N)$ which has no zeroes or poles in $\mathbb{H}$, this shows that $g$ is constant. Examining (63) for $a / c=1 / 0=\infty$ we have $A=\left(\begin{array}{ll}1 & 0 \\ 0 & 1\end{array}\right), d=N$ and $e_{1 / N}=e_{\infty}=e$. So if $G=g \circ e$ and $F=f \circ e$ then $(63)$ becomes

$$
G=p^{1-k} \xi_{0} \frac{F \mid T_{p}}{F}
$$


Since $g$ is constant this says that $F \mid T_{p}=\lambda(p) F$ for some complex constant $\lambda(p)$ and the theorem stated in the introduction is now proven.

7. Observations. We set

$$
A_{\delta, d}=24 \times \operatorname{Ord}_{\Gamma_{N}(N)}\left(\eta \circ \delta ; \frac{1}{d}\right)=\frac{N}{\left(N, d^{2}\right)} \frac{(d, \delta)^{2}}{d}
$$

so that

$$
C(d)=\sum_{\delta \mid N} A_{\delta, d} r_{\delta}
$$

The matrix $\left(A_{\delta . d}\right)$ is an invertible matrix with positive integral entries, so the number of integral solutions $\left(r_{\delta} ; \delta \mid N\right)$ for the system of inequalities

$$
0 \leq C(d) \leq 24 \text { for all } d \mid N
$$

is finite for any fixed $N$. The inverse of $\left(A_{\delta, d}\right)$ can be exhibited explicitly as $\left(B_{d, \delta}\right)$, where

$$
B_{d, \delta}=\frac{\left(N, d^{2}\right)(d, \delta)^{2}}{d^{2} \delta N \prod_{p \mid N}\left(1-\frac{1}{p^{2}}\right)} \times \sum_{\alpha|(d, \delta N)| d, \delta \mid} \frac{\mu\left(\frac{\alpha d}{(d, \delta)}\right) \mu\left(\frac{\alpha \delta}{(d, \delta)}\right)}{\alpha^{2}},
$$

where $\mu$ denotes the Möbius mu-function. (This appeared in a different form in Biagioli [4, p. 281, Proposition 3.3]). Multiplying (65) by $B_{d, \delta}$ and summing over $d$ we see that

$$
24 \times \sum_{B_{d, \delta} \leq 0} B_{d, \delta}<r_{\delta}<24 \times \sum_{B_{d, \delta} \geq 0} B_{d, \delta},
$$

which gives an effective bound for the possible values of $r_{\delta}$.

As $N$ runs over all prime numbers Morris Newman [12] found that there was only a finite number of solutions. It should be possible to determine whether the number of solutions remains finite when $N$ runs over all positive integers; and whether the number of solution is finite or not it would be good to have a more convenient way to determine them; using (66) and checking (65) for all truples $\left(r_{\delta} ; \delta \mid N\right)$ in that range seems inefficient.

Once a specific form $f$ has been found for which $F$ is an eigenform of all $T_{p}$ for which $p \nmid N$ and $p \equiv 1(\bmod E)$, one can use the approach of Gordon and Sinor [6] and Gordon and Hughes [5] to find completing forms $f_{1}, f_{2}, \ldots, f_{n}$ so that the sum $F_{1}+F_{2}+\ldots+F_{n}$ is an eigenform for all $T_{p}$ for which $p \nmid N$. The drawback of their approach is that it doesn't guarantee finding the completing forms, and there is no specific guide as to how to choose the function they refer to as an auxiliary function. It would be good to know when their technique works and what the auxiliary function should be in the general setting.

In [12], [13], [5], and [6] some conclusions were drawn about multiplicative properties and about the lacunarity of the Fourier series of the simultaneous eigenforms found there. Similar conclusions can be drawn for any further eigenforms found by these techniques. 


\section{REFERENCES}

1. Bruce C. Berndt, Ramanujan's Notebooks, Part III (Springer-Verlag, 1991).

2. Bruce C. Berndt, Anthony J. F. Biagioli and James, Purtilo, Ramanujan's modular equations of 'large' prime degree, J. Indian Math. Soc. 51 (1987), 75-110.

3. Anthony J. F. Biagioli, Products of Powers of Transforms of the Dedekind Eta-function, thesis (University of Wisconsin-Madison, 1982).

4. Anthony J. F. Biagioli, The construction of modular forms as products of transforms of the Dedekind eta-function, Acta Arith. 54 (1990), 273-300.

5. Basil Gordon and Kim Hughes, Multiplicative properties of eta-products II, to appear.

6. Basil Gordon and Dale Sinor, Multiplicative properties of eta-products, Springer Lecture Notes in Mathematics 1395 (1988), 173-200. 1970).

7. Marvin I. Knopp, Modular Forms in Analytic Number Theory, (Markham Publ. Co., 1964).

8. Joseph Lehner, Discontinuous Groups and Automorphic Functions, (Amer. Math. Soc. $1-80$.

9. G. Ligozat, Courbes modulaaires de genre 1, Bull. Soc. Math. France, Mém. 43 (1972),

10. Morris Newman, Construction and application of a certain class of modular functions, Proc. London Math. Soc. (3) 7 (1956), 334-350.

11. Morris Newman, Construction and application of a certain class of modular functions II, Proc. London Math. Soc. (3) 9 (1959), 373-387.

12. Morris Newman, Modular forms whose coefficients possess multiplicative properties, Ann. Math. 70 (1959), 478-489.

13. Morris Newman, Modular forms whose coefficients possess multiplicative properties II, Ann. Math. 75 (1962), 242-250.

14. Robert Rankin, Modular Forms and Functions (Cambridge University Press, 1977).

15. Bruno Schoeneberg, Elliptic Modular Functions (Springer-Verlag, 1974).

University of Central Arkansas

Conway, Arkansas, USA

AND

Pennsylvania State University, Berks Campus

Reading, Pennsylvania, USA 